\title{
FINITE DIMENSIONAL HILBERT SPACES ARE COMPLETE FOR DAGGER COMPACT CLOSED CATEGORIES*
}

\author{
PETER SELINGER
}

Department of Mathematics and Statistics, Dalhousie University, Halifax, Nova Scotia, Canada

e-mail address: selinger@mathstat.dal.ca

\begin{abstract}
We show that an equation follows from the axioms of dagger compact closed categories if and only if it holds in finite dimensional Hilbert spaces.
\end{abstract}

\section{INTRODUCTION}

Hasegawa, Hofmann, and Plotkin recently showed that the category of finite dimensional vector spaces over any fixed field $k$ of characteristic 0 is complete for traced symmetric monoidal categories [3. What this means is that an equation holds in all traced symmetric monoidal categories if and only if it holds in finite dimensional vector spaces. The authors also noted that it is a direct corollary, via Joyal, Street, and Verity's "Int"-construction [4, that finite dimensional vector spaces are also complete for compact closed categories. The present paper makes two contributions: (1) we simplify the proof of Hasegawa, Hofmann, and Plotkin's result, and (2) we extend it to show that finite dimensional Hilbert spaces are complete for dagger traced symmetric monoidal categories (and hence for dagger compact closed categories).

The paper is organized as follows. We assume the reader knows the definition of a dagger compact closed category [1, 7], and has at least an informal understanding of their graphical language [8]. In Section 2, we state the main result without further ado. Sections 3 and 4 are devoted to an informal, but hopefully comprehensible, explanation of the proof. For the reader who is interested in details, full technical definitions and proofs (including a formal definition of the graphical language and isomorphism of diagrams) appear in Section 5. Section [6] discusses how to generalize the result to fields other than the complex numbers, gives counterexamples for some possible strengthenings of completeness, and concludes with an open problem.

1998 ACM Subject Classification: F.3.2, F.4.1.

Key words and phrases: Dagger compact closed categories, Hilbert spaces, completeness.

* An extended abstract of this work has appeared in Proceedings of the 5th International Workshop on Quantum Physics and Logic (QPL 2008), Electronic Notes in Theoretical Computer Science 270(1):113-119, 2011.

Research supported by NSERC..

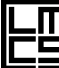

DOI:10.2168/LMCS-8 (3:06) 2012

(C) P. Selinger

(c) Creative Commons 


\section{Statement of the main Result}

For a definition of dagger compact closed categories, their term language, and their graphical language, see [1, 7, 8]. We also use the concept of a dagger traced monoidal category [8], which is a dagger symmetric monoidal category with a trace operation [4 satisfying $\operatorname{Tr}_{U, V}^{X}(f)^{\dagger}=\operatorname{Tr}_{V, U}^{X}\left(f^{\dagger}\right)$. We note that every dagger compact closed category is also dagger traced monoidal; conversely, by Joyal, Street, and Verity's "Int" construction, every dagger traced monoidal category can be fully embedded in a dagger compact closed category.

We will make use of the soundness and completeness of the graphical representation, specifically of the following result:

Theorem 2.1 ([7]). A well-typed equation between morphisms in the language of dagger compact closed categories follows from the axioms of dagger compact closed categories if and only if it holds, up to graph isomorphism, in the graphical language.

An analogous result also holds for dagger traced monoidal categories [8, Thm. 7.12]. The goal of this paper is to prove the following:

Theorem 2.2. Let $M, N: A \rightarrow B$ be two terms in the language of dagger compact closed categories. Suppose that $\llbracket M \rrbracket=\llbracket N \rrbracket$ for every possible interpretation (of object variables as spaces and morphism variables as linear maps) in finite dimensional Hilbert spaces. Then $M=N$ holds in the graphical language (and therefore, holds in all dagger compact closed categories).

\section{REDUCTIONS}

Before attempting to prove Theorem 2.2, we reduce the statement to something simpler. By arguments analogous to those of Hasegawa, Hofmann, and Plotkin [3], it suffices without loss of generality to consider terms $M, N$ that satisfy some additional conditions. The additional conditions are:

- It suffices to consider terms whose graphical representation does not contain any "trivial cycles". Trivial cycles are connected components of a diagram that do not contain any morphism variables. They can arise from the trace of an identity morphism. The restriction is without loss of generality because if $M, N$ have different numbers or types of trivial cycles, they can be easily separated in Hilbert spaces. Details are given in Lemma 5.11 below. We say that a diagram is simple if it contains no trivial cycles, and a term is simple if its associated diagram is simple.

- We may assume that $M, N: I \rightarrow I$, i.e., that both the domain and codomain of $M$ and $N$ are the tensor unit. Such terms are called closed. This simplification is justified in Lemma 5.12 below.

- It suffices to consider terms $M, N$ in the language of dagger traced monoidal categories. Namely, by Joyal, Street, and Verity's "Int"-construction [4], every statement about dagger compact closed categories can be translated to an equivalent statement about dagger traced monoidal categories. Informally, this is done by eliminating occurrences of the *-operation: one replaces every morphism variable such as $f: A^{*} \otimes B \otimes C^{*} \rightarrow D^{*} \otimes E$ by an equivalent new morphism variable such as $f^{\prime}: B \otimes D \rightarrow A \otimes C \otimes E$ that does not use the $*$-operation. Details are given in Lemma 5.13 below. 


\section{INFORMAL OUTLINE OF THE RESUlT}

The formal statement and proof of Theorem 2.2 requires a fair amount of notation. Nevertheless, the main idea is simple, and is perhaps best illustrated in an example. We thus start by giving an informal explanation of the proof in this section. The full technical proof, including formal definitions of the graphical language and isomorphism of diagrams, is given in Section 5 .

4.1. Signatures, diagrams, and interpretations. We assume given a set of object variables, denoted $A, B$ etc., and a set of morphism variables, denoted $f, g$ etc. A sort $\boldsymbol{A}$ is a finite sequence of object variables. We usually write $A_{1} \otimes \ldots \otimes A_{n}$ for an $n$-element sequence, and $I$ for the empty sequence. We assume that each morphism variable $f$ is assigned two fixed sorts, called its domain $\boldsymbol{A}$ and codomain $\boldsymbol{B}$ respectively, and we write $f: \boldsymbol{A} \rightarrow \boldsymbol{B}$. We further require a fixed-point free involution $(-)^{\dagger}$ on the set of morphism variables, such that $f^{\dagger}: \boldsymbol{B} \rightarrow \boldsymbol{A}$ when $f: \boldsymbol{A} \rightarrow \boldsymbol{B}$.

The collection of object variables and morphism variables, together with the domain and codomain information and the dagger operation is called a signature $\Sigma$ of dagger monoidal categories.

Graphically, we represent a morphism variable $f: A_{1} \otimes \ldots \otimes A_{n} \rightarrow B_{1} \otimes \ldots \otimes B_{m}$ as a box

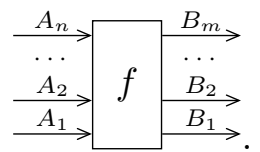

The wires on the left are called the inputs of $f$, and the wires on the right are called its outputs. Note that each box is labeled by a morphism variable, and each wire is labeled by an object variable.

A (simple closed dagger traced symmetric monoidal) diagram over a signature $\Sigma$ consists of zero or more boxes of the above type, all of whose wires have been connected in pairs, such that each connection is between the output wire of some box and the input wire of some (possibly the same, possibly another) box. Here is an example of a diagram $N$ over the signature given by $f: B \rightarrow A \otimes A$ and $g: A \otimes B \rightarrow B \otimes A$.

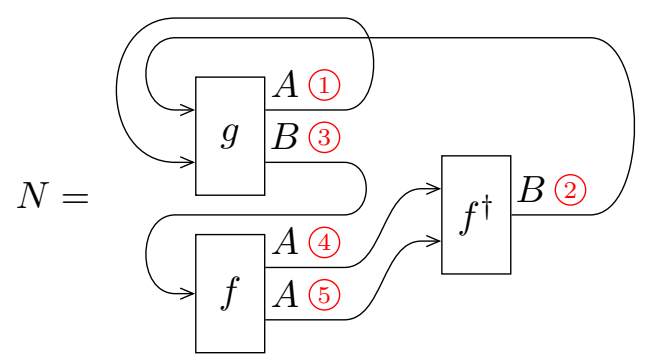

In the illustration, we have numbered the wires 1 to 5 to aid the exposition below; note that this numbering is not formally part of the diagram.

An interpretation of a signature in finite-dimensional Hilbert spaces consists of the following data: for each object variable $A$, a chosen finite-dimensional Hilbert space $\llbracket A \rrbracket$, and for each morphism variable $f: A_{1} \otimes \ldots \otimes A_{n} \rightarrow B_{1} \otimes \ldots \otimes B_{m}$, a chosen linear map $\llbracket f \rrbracket: \llbracket A_{1} \rrbracket \otimes \ldots \otimes \llbracket A_{n} \rrbracket \rightarrow \llbracket B_{1} \rrbracket \otimes \ldots \otimes \llbracket B_{m} \rrbracket$, such that $\llbracket f^{\dagger} \rrbracket=\llbracket f \rrbracket^{\dagger}$. 
The denotation of a diagram $M$ under a given interpretation is a scalar that is defined by the usual "summation over internal indices" formula. For example, the denotation of the above diagram $N$ is:

$$
\llbracket N \rrbracket=\sum_{a_{1}, b_{2}, b_{3}, a_{4}, a_{5}} \llbracket g \rrbracket_{a_{1}, b_{2}}^{b_{3}, a_{1}} \cdot \llbracket f \rrbracket_{b_{3}}^{a_{5}, a_{4}} \cdot \llbracket f^{\dagger} \rrbracket_{a_{5}, a_{4}}^{b_{2}} \cdot
$$

Here $a_{1}, a_{4}, a_{5}$ range over some orthonormal basis of $\llbracket A \rrbracket, b_{2}, b_{3}$ range over some orthonormal basis of $\llbracket B \rrbracket$, and $\llbracket f \rrbracket_{b_{3}, a_{4}}^{a_{5}}$ stands for the matrix entry $\left\langle a_{5} \otimes a_{4} \mid \llbracket f \rrbracket\left(b_{3}\right)\right\rangle$. As is well-known, this denotation is independent of the choice of orthonormal basis [2, 6, 9].

4.2. Proof sketch. By the reductions in Section 3 , Theorem 2.2 is a consequence of the following lemma:

Lemma 4.1 (Relative completeness). Let $M$ be a (simple closed dagger traced monoidal) diagram. Then there exists an interpretation $\llbracket-\rrbracket_{M}$ in finite dimensional Hilbert spaces, depending only on $M$, such that for all $N, \llbracket N \rrbracket_{M}=\llbracket M \rrbracket_{M}$ holds if and only if $N$ and $M$ are isomorphic diagrams.

Clearly, the right-to-left implication is trivial, for if $N$ and $M$ are isomorphic diagrams, then $\llbracket N \rrbracket=\llbracket M \rrbracket$ holds under every interpretation; their corresponding summation formulas differ at most by a reordering of summands and factors. It is therefore the left-to-right implication that must be proved.

The general proof of this lemma requires quite a bit of notation, as well as more careful definitions than we have given above. A full proof appears in Section 5 below. Here, we illustrate the proof technique by means of an example.

Take the same signature as above, and suppose $M$ is the following diagram:

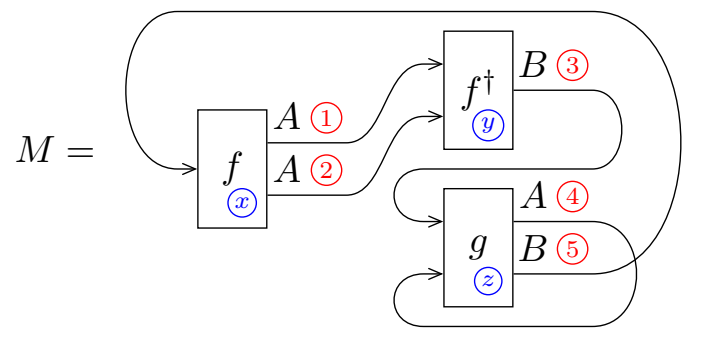

Again, we have numbered the wires from 1 to 5, and this time, we have also numbered the boxes $x, y$, and $z$.

We must now construct the interpretation required by the Lemma. It is given as follows. Define $\llbracket A \rrbracket_{M}$ to be a 3 -dimensional Hilbert space with orthonormal basis $\left\{A_{1}, A_{2}, A_{4}\right\}$. Define $\llbracket B \rrbracket_{M}$ to be a 2 -dimensional Hilbert space with orthonormal basis $\left\{B_{3}, B_{5}\right\}$. Note that the names of the basis vectors have been chosen to suggest a correspondence between basis vectors of $\llbracket A \rrbracket_{M}$ and wires labeled $A$ in the diagram $M$, and similarly for $\llbracket B \rrbracket_{M}$.

Let $x, y$, and $z$ be three algebraically independent transcendental complex numbers. This means that $x, y, z$ do not satisfy any polynomial equation $p(x, y, z, \bar{x}, \bar{y}, \bar{z})=0$ with rational coefficients, unless $p \equiv 0$.

Define three linear maps $F_{x}: \llbracket B \rrbracket_{M} \rightarrow \llbracket A \rrbracket_{M} \otimes \llbracket A \rrbracket_{M}, F_{y}: \llbracket A \rrbracket_{M} \otimes \llbracket A \rrbracket_{M} \rightarrow \llbracket B \rrbracket_{M}$, and $F_{z}: \llbracket A \rrbracket_{M} \otimes \llbracket B \rrbracket_{M} \rightarrow \llbracket B \rrbracket_{M} \otimes \llbracket A \rrbracket_{M}$ as follows. We give each map by its matrix 
representation in the chosen basis.

$$
\begin{aligned}
& \left(F_{x}\right)_{i}^{j k}=\left\{\begin{array}{l}
x \text { if } i=B_{5}, j=A_{2}, \text { and } k=A_{1}, \\
0 \text { otherwise }
\end{array}\right. \\
& \left(F_{y}\right)_{i j}^{k}=\left\{\begin{array}{l}
y \text { if } i=A_{2}, j=A_{1}, \text { and } k=B_{3}, \\
0 \text { otherwise }, \\
z \text { if } i=A_{4}, j=B_{3}, k=B_{5}, \text { and } l=A_{4}, \\
0 \text { otherwise. }
\end{array}\right.
\end{aligned}
$$

It is hopefully obvious how each of these linear functions is derived from the diagram $M$ : each matrix contains precisely one non-zero entry, whose position is determined by the numbering of the input and output wires of the corresponding box in $M$.

The interpretations of $f$ and $g$ are then defined as follows:

$$
\llbracket f \rrbracket_{M}=F_{x}+F_{y}^{\dagger}, \quad \llbracket g \rrbracket_{M}=F_{z} .
$$

Note that we have taken the adjoint of the matrix $F_{y}$, due to the fact that the corresponding box was labeled $f^{\dagger}$. This finishes the definition of the interpretation $\llbracket-\rrbracket_{M}$. It can be done analogously for any diagram $M$.

The reader may wonder why we didn't simply define $\llbracket f \rrbracket_{M}=F_{x}$ and $\llbracket f^{\dagger} \rrbracket_{M}=F_{y}$ independently of each other. The reason is of course that an interpretation must satisfy $\llbracket f^{\dagger} \rrbracket_{M}=\llbracket f \rrbracket_{M}^{\dagger}$.

To prove the condition of the Lemma, we first observe that the interpretation $\llbracket N \rrbracket_{M}$ of any diagram $N$ is given by a summation formula analogous to (4.1). Moreover, from the definition of the interpretation $\llbracket-\rrbracket_{M}$, it immediately follows that the scalar $\llbracket N \rrbracket_{M}$ can be (uniquely) expressed as a polynomial $p(x, y, z, \bar{x}, \bar{y}, \bar{z})$ with integer coefficients in the variables $x, y, z$ and their complex conjugates. We note in passing that this polynomial is homogeneous, and its degree is equal to the number of boxes in $N$.

We claim that the coefficient of $p$ at $x y z$ is non-zero if and only if $N$ is isomorphic to $M$. The proof is a direct calculation, using (4.1) and the definition of $\llbracket-\rrbracket_{M}$. Essentially, any non-zero contribution to $x y z$ in the summation formula must come from a choice of a basis vector $A_{\phi(w)}$ of $\llbracket A \rrbracket_{M}$ for each wire $w$ labeled $A$ in $N$, and a choice of a basis vector $B_{\phi(w)}$ of $\llbracket B \rrbracket_{M}$ for each wire $w$ labeled $B$ in $N$, together with a bijection $\psi$ between the boxes of $N$ and the set $\{x, y, z\}$; moreover, the contribution can only be non-zero if the choice of basis vectors is "compatible" with the bijection $\psi$. Compatibility amounts precisely to the requirement that the maps $\phi$ and $\psi$ determine a graph isomorphism from $N$ to $M$. For example, in the calculation of $\llbracket N \rrbracket_{M}$ according to equation (4.1), the only non-zero contribution to $x y z$ in $p$ comes from the assignment $a_{1} \mapsto A_{4}, b_{2} \mapsto B_{3}, b_{3} \mapsto B_{5}, a_{4} \mapsto A_{1}$, and $a_{5} \mapsto A_{2}$, which corresponds exactly to the (in this case unique) isomorphism from $N$ to $M$.

In fact, we get a stronger result: the integer coefficient of $p$ at $x y z$ is equal to the number of different isomorphisms between $N$ and $M$ (usually 0 or 1 , but it could be higher if $M$ has non-trivial automorphisms).

\section{Technical Development}

\subsection{Signatures and diagrams.}


Definition 5.1. (Signature) A signature of dagger monoidal categories is a quintuple $\Sigma=\langle\mathrm{Obj}$, Mor, dom, cod, $\dagger\rangle$ consisting of:

- a set Obj of object variables, denoted $A, B, C, \ldots$;

- a set Mor of morphism variables, denoted $f, g, h, \ldots$;

- functions dom, cod : Mor $\rightarrow \mathrm{Obj}^{*}$, called the domain and codomain functions, respectively, where $\mathrm{Obj}^{*}$ is the set of finite sequences of object variables;

- an operation $\dagger:$ Mor $\rightarrow$ Mor, such that for all $f \in$ Mor, $f^{\dagger \dagger}=f, f^{\dagger} \neq f$, $\operatorname{dom} f^{\dagger}=\operatorname{cod} f$, and $\operatorname{cod} f^{\dagger}=\operatorname{dom} f$.

As before, we write a sequence of $n$ object variables as $A_{1} \otimes \ldots \otimes A_{n}$, or as $\boldsymbol{A}$, and we write $|\boldsymbol{A}|=n$ for the length of a sequence. We write $f: \boldsymbol{A} \rightarrow \boldsymbol{B}$ if $\operatorname{dom} f=\boldsymbol{A}$ and $\operatorname{cod} f=\boldsymbol{B}$.

Definition 5.2. (Diagram) A (simple closed dagger traced symmetric monoidal) diagram $M=\left\langle W^{M}, B^{M}, \ell_{\mathrm{w}}^{M}, \ell_{\mathrm{b}}^{M}, \theta_{\mathrm{in}}^{M}, \theta_{\mathrm{out}}^{M}\right\rangle$ over a signature $\Sigma$ consists of the following:

- a finite set $W^{M}$ of wires;

- a finite set $B^{M}$ of boxes;

- a pair of labeling functions $\ell_{\mathrm{w}}^{M}: W^{M} \rightarrow$ Obj and $\ell_{\mathrm{b}}^{M}: B^{M} \rightarrow$ Mor;

- a pair of bijections $\theta_{\text {in }}^{M}:$ Inputs $^{M} \rightarrow W^{M}$ and $\theta_{\text {out }}^{M}$ : Outputs ${ }^{M} \rightarrow W^{M}$, where

$$
\begin{aligned}
& \text { Inputs }^{M}=\left\{(i, b)\left|b \in B^{M}, n=\right| \operatorname{dom}\left(\ell_{\mathrm{b}}^{M}(b)\right) \mid, 1 \leqslant i \leqslant n\right\}, \\
& \text { Outputs }^{M}=\left\{(b, j)\left|b \in B^{M}, m=\right| \operatorname{cod}\left(\ell_{\mathrm{b}}^{M}(b)\right) \mid, 1 \leqslant j \leqslant m\right\} .
\end{aligned}
$$

Moreover, a diagram is required to satisfy the following typing conditions:

- whenever $b \in B^{M}, f=\ell_{\mathrm{b}}^{M}(b), \boldsymbol{A}=\operatorname{dom} f,(i, b) \in \operatorname{Inputs}^{M}, \theta_{\mathrm{in}}^{M}(i, b)=w$, then $\ell_{\mathrm{w}}^{M}(w)=$ $A_{i}$, and

- whenever $b \in B^{M}, f=\ell_{\mathrm{b}}^{M}(b), \boldsymbol{B}=\operatorname{cod} f,(b, j) \in \operatorname{Outputs}^{M}, \theta_{\text {out }}^{M}(b, j)=w$, then $\ell_{\mathrm{w}}^{M}(w)=B_{j}$.

Informally, $(i, b)$ represents the $i$ th input of box $b,(b, j)$ represents the $j$ th output of box $b$, and the bijections $\theta_{\mathrm{in}}^{M}$ and $\theta_{\text {out }}^{M}$ determine which wires are attached to which inputs and outputs, respectively. The labeling functions assign an object variable to each wire and a morphism variable to each box, and the typing conditions ensure that the sort of each wire matches the sort of each box it is attached to.

Definition 5.3. (Isomorphism) An isomorphism of diagrams $N, M$ is given by a pair of bijections $\phi: W^{N} \rightarrow W^{M}$ and $\psi: B^{N} \rightarrow B^{M}$, commuting with the labeling functions and with $\theta_{\text {in }}$ and $\theta_{\text {out }}$. Explicitly, this means that for all $w \in W^{N}$ and $b \in B^{N}$, and all $i \leqslant\left|\operatorname{dom}\left(\ell_{\mathrm{b}}^{N}(b)\right)\right|$ and $j \leqslant\left|\operatorname{cod}\left(\ell_{\mathrm{b}}^{N}(b)\right)\right|$,

$$
\begin{aligned}
\ell_{\mathrm{w}}^{M}(\phi(w)) & =\ell_{\mathrm{w}}^{N}(w), \\
\ell_{\mathrm{b}}^{M}(\psi(b)) & =\ell_{\mathrm{b}}^{N}(b), \\
\theta_{\mathrm{in}}^{M}(i, \psi(b)) & =\phi\left(\theta_{\mathrm{in}}^{N}(i, b)\right), \\
\theta_{\mathrm{out}}^{M}(\psi(b), j) & =\phi\left(\theta_{\text {out }}^{N}(b, j)\right) .
\end{aligned}
$$

Lemma 5.4. In the definition of isomorphism, the condition that $\phi$ is a bijection is redundant. 
Proof. The bijection $\psi: B^{N} \rightarrow B^{M}$ induces a bijection $\hat{\psi}: \operatorname{Inputs}^{N} \rightarrow \operatorname{Inputs}^{M}$, defined by $\hat{\psi}(b, j)=(\psi(b), j)$. Equation (5.4) is then equivalent to the commutativity of this diagram:

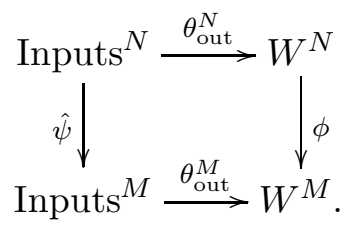

Since the top, bottom, and left arrows are bijections, so is the right arrow.

\subsection{Interpretation in finite dimensional Hilbert spaces.}

Definition 5.5. (Interpretation) Let $\Sigma$ be a signature. An interpretation $\llbracket-\rrbracket$ of $\Sigma$ in the category of finite dimensional Hilbert spaces assigns to each object variable $A \in$ Obj a finite dimensional Hilbert space $\llbracket A \rrbracket$, and to each morphism variable $f: \boldsymbol{A} \rightarrow \boldsymbol{B}$ a linear map $\llbracket f \rrbracket: \llbracket A_{1} \rrbracket \otimes \ldots \otimes \llbracket A_{n} \rrbracket \rightarrow \llbracket B_{1} \rrbracket \otimes \ldots \otimes \llbracket B_{m} \rrbracket$, such that for all $f, \llbracket f^{\dagger} \rrbracket=\llbracket f \rrbracket^{\dagger}$.

We sometimes write $\llbracket \boldsymbol{A} \rrbracket$ for $\llbracket A_{1} \rrbracket \otimes \ldots \otimes \llbracket A_{n} \rrbracket$.

Definition 5.6. (Denotation) Given a signature $\Sigma$, a diagram $N$, and an interpretation $\llbracket-\rrbracket$. Fix an orthogonal basis Basis $_{A}$ for each space $\llbracket A \rrbracket$. An indexing of $N$ is a function $\phi \in \prod_{w \in W^{N}} \operatorname{Basis}_{\ell_{\mathrm{w}}^{N}(w)}$, i.e., a choice of a basis element $\phi(w) \in \operatorname{Basis}_{\ell_{\mathrm{w}}^{N}(w)}$ for every wire $w \in W^{N}$. The set of indexings is written $\operatorname{Idx}^{N}$. Then to each pair of an indexing $\phi$ and a box $b$, we assign a matrix entry

$$
b(\phi)=\llbracket f \rrbracket_{\phi\left(\theta_{\text {in }}^{N}(1, b)\right), \ldots, \phi\left(\theta_{\text {in }}^{N}(n, b)\right)}^{\phi\left(\theta_{0}^{N}(b, 1)\right), \ldots, \phi\left(\theta^{N}(b, m)\right)}
$$

where $f=\ell_{\mathrm{b}}^{N}(b): A_{1} \otimes \ldots \otimes A_{m} \rightarrow B_{1} \otimes \ldots \otimes B_{m}$. As before, we have written $F_{y_{1}, \ldots, y_{n}}^{x_{1}, \ldots, x_{m}}$ for the inner product $\left\langle x_{1} \otimes \ldots \otimes x_{m} \mid F\left(y_{1} \otimes \ldots \otimes y_{n}\right)\right\rangle$. The denotation of $N$ is a scalar $\llbracket N \rrbracket$ defined as follows:

$$
\llbracket N \rrbracket=\sum_{\phi \in \mathrm{Idx}^{N}} \prod_{b \in B^{N}} b(\phi) .
$$

Remark 5.7. The definition of $\llbracket N \rrbracket$ is independent of the chosen bases. In fact, the formula for $\llbracket N \rrbracket$ is just the usual formula for the summation over internal indices. It is the same as equation (4.1), expressed in the general context.

Remark 5.8. The graphical language can be interpreted in any dagger compact closed category [7, 8]. In the case of FinHilb, the general interpretation coincides with the one given here.

5.3. The $M$-interpretation. We are now in a position to give a formal proof of relative completeness (Lemma 4.1). Fix a diagram $M$. We will define a particular interpretation $\llbracket-\rrbracket_{M}$, called the $M$-interpretation, with the property that $\llbracket N \rrbracket_{M}=\llbracket M \rrbracket_{M}$ if and only if $N$ and $M$ are isomorphic.

A family $\left\{\xi_{1}, \ldots, \xi_{k}\right\}$ of transcendental complex numbers is algebraically independent if for every polynomial $p$ with rational coefficients, $p\left(\xi_{1}, \ldots, \xi_{k}, \bar{\xi}_{1}, \ldots, \bar{\xi}_{k}\right)=0$ implies $p \equiv 0$. We choose a family of algebraically independent transcendental numbers $\left\{\xi_{b} \mid b \in B^{M}\right\}$. This is possible because $\mathbb{R}$ is a field extension of infinite transcendence degree over $\mathbb{Q}[5]$, 
P. SELINGER

and because the complex numbers $a_{1}+i b_{1}, \ldots, a_{k}+i b_{k}$ are algebraically independent in the above sense if and only if $a_{1}, \ldots, a_{k}, b_{1}, \ldots, b_{k}$ are algebraically independent real numbers.

For each object variable $A$, let $W_{A}^{M}$ be the set of all wires of $M$ that are labeled $A$, and for each morphism variable $f$, let $B_{f}^{M}$ be the set of boxes of $M$ that are labeled $f$. In symbols,

$$
\begin{aligned}
& W_{A}^{M}=\left\{w \in W^{M} \mid \ell_{\mathrm{w}}^{M}(w)=A\right\}, \\
& B_{f}^{M}=\left\{b \in B^{M} \mid \ell_{\mathrm{b}}^{M}(b)=f\right\} .
\end{aligned}
$$

Then the $M$-interpretation $\llbracket-\rrbracket_{M}$ is defined as follows. For each $A$, let $\llbracket A \rrbracket_{M}$ be a Hilbert space with orthonormal basis $W_{A}^{M}$. Suppose $f: \boldsymbol{A} \rightarrow \boldsymbol{B}$ is a morphism variable, and consider some $f$-labeled box $b \in B_{f}^{M}$. We define a linear map $F_{b}: \llbracket \boldsymbol{A} \rrbracket_{M} \rightarrow \llbracket \boldsymbol{B} \rrbracket_{M}$ by its matrix entries

$$
\left(F_{b}\right)_{w_{1}, \ldots, w_{n}}^{w_{1}^{\prime}, \ldots, w_{m}^{\prime}}= \begin{cases}\xi_{b} \text { if } w_{i}=\theta_{\mathrm{in}}^{M}(i, b) \text { and } w_{j}^{\prime}=\theta_{\text {out }}^{M}(b, j) \text { for all } i, j \\ 0 \text { otherwise }\end{cases}
$$

where $w_{i} \in W_{A_{i}}^{M}$ and $w_{j}^{\prime} \in W_{B_{j}}^{M}$ range over basis vectors. Finally, we define

$$
\llbracket f \rrbracket_{M}=\sum_{b \in B_{f}^{M}} F_{b}+\sum_{b \in B_{f^{\dagger}}^{M}} F_{b}^{\dagger} .
$$

5.4. Proof of relative completeness. We must prove that the $M$-interpretation satisfies relative completeness (Lemma 4.1). First, we compute the $M$-interpretation of any diagram $N$. By (5.6) and (5.5), we have

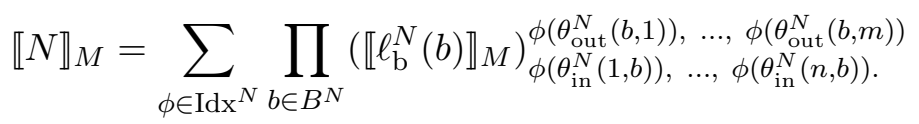

Using (5.8), it follows that

$$
\begin{aligned}
\llbracket N \rrbracket_{M} & =\sum_{\phi \in \operatorname{Idx}^{N}} \prod_{b \in B^{N}} \sum_{b^{\prime} \in B_{\ell_{\mathrm{b}}^{N}(b)}^{M}}\left(F_{b^{\prime}}\right)_{\phi\left(\theta_{\mathrm{in}}^{N}(1, b)\right), \ldots, \phi\left(\theta_{\mathrm{in}}^{N}(n, b)\right)}^{\phi\left(\theta_{\text {out }}^{N}(b, 1)\right), \ldots, \phi\left(\theta^{N}(b, m)\right)} \\
& +\sum_{\phi \in \operatorname{Idx}^{N}} \prod_{b \in B^{N}} \sum_{b^{\prime} \in B_{\ell_{\mathrm{b}}^{N}(b)^{\dagger}}^{M}}\left(F_{b^{\prime}}^{\dagger}\right)_{\phi\left(\theta_{\mathrm{in}}^{N}(1, b)\right), \ldots, \phi\left(\theta_{\mathrm{in}}^{N}(n, b)\right) .}^{\phi\left(\theta_{\text {out }}^{N}(b, 1)\right), \ldots, \phi\left(\theta_{\text {out }}^{N}(b, m)\right)}
\end{aligned}
$$

Now, using (5.7) and the definition of $\dagger$, we obtain the following explicit summation formula:

$$
\begin{aligned}
& \llbracket N \rrbracket_{M}=\sum_{\phi \in \mathrm{Idx}^{N}} \prod_{b \in B^{N}} \sum_{b^{\prime} \in B_{\ell_{\mathrm{b}}^{N}(b)}^{M}}\left\{\begin{array}{l}
\xi_{b^{\prime}} \text { if } \phi\left(\theta_{\mathrm{in}}^{N}(i, b)\right)=\theta_{\mathrm{in}}^{M}\left(i, b^{\prime}\right) \text { and } \\
\phi\left(\theta_{\text {out }}^{N}(b, j)\right)=\theta_{\text {out }}^{M}\left(b^{\prime}, j\right) \text { for all } i, j, \\
0 \quad \begin{array}{l}
\text { otherwise, }
\end{array}
\end{array}\right.
\end{aligned}
$$

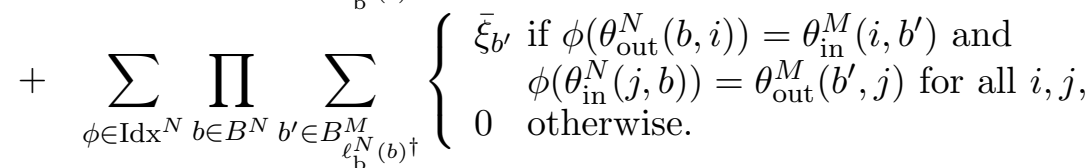

We note at this point that $\llbracket N \rrbracket_{M}$ can be (uniquely) written as a polynomial with nonnegative integer coefficients in the variables $\left\{\xi_{b}, \bar{\xi}_{b} \mid b \in B^{M}\right\}$. Moreover, this polynomial is homogeneous. 
By definition, $b^{\prime} \in B_{\ell_{\mathrm{b}}^{N}(b)}^{M}$ if and only if $\ell_{\mathrm{b}}^{M}\left(b^{\prime}\right)=\ell_{\mathrm{b}}^{N}(b)$, and $b^{\prime} \in B_{\ell_{\mathrm{b}}^{N}(b)^{\dagger}}^{M}$ if and only if $\ell_{\mathrm{b}}^{M}\left(b^{\prime}\right)=\ell_{\mathrm{b}}^{N}(b)^{\dagger}$. The sets $B_{\ell_{\mathrm{b}}^{N}(b)}^{M}$ and $B_{\ell_{\mathrm{b}}^{N}(b)^{\dagger}}^{M}$ are disjoint for each given $b$, since $\dagger$ is fixed-point free. We can therefore rewrite the summation as:

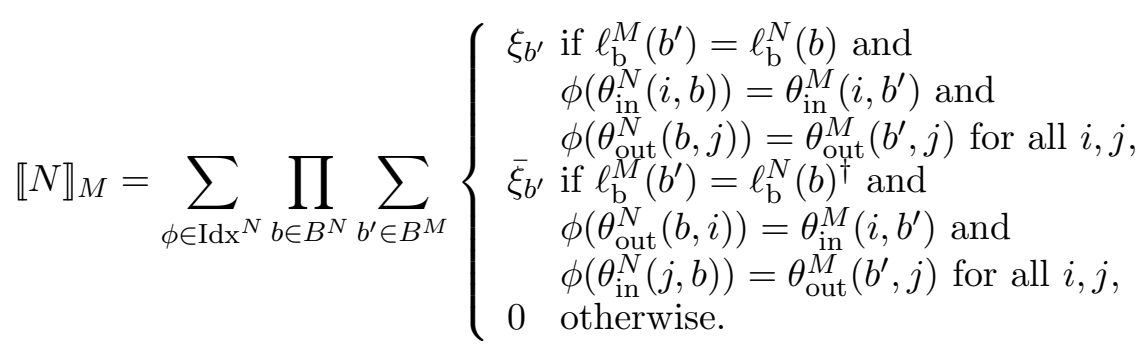

Finally, we use the distributive law to exchange the order of addition and multiplication.

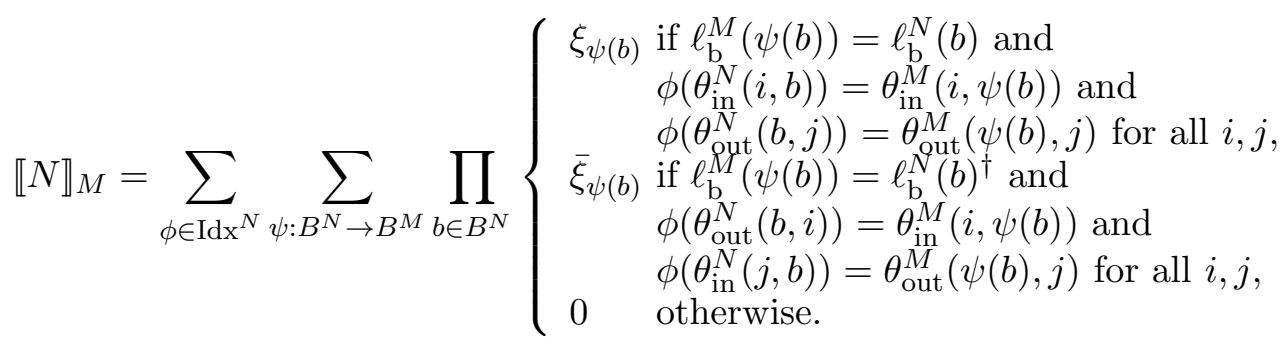

Now consider a fixed $\phi \in \operatorname{Idx}^{N}$ and fixed $\psi: B^{N} \rightarrow B^{M}$. We claim that the product $\prod_{b \in B^{N}}(\ldots)$ in (5.9) is equal to $\prod_{b \in B^{M}} \xi_{b}$ if and only if the pair of maps $(\phi, \psi)$ forms an isomorphism of diagrams from $N$ to $M$. Indeed, the product in question is equal to $\prod_{b \in B^{M}} \xi_{b}$ if and only if $\psi$ is a bijection and the first side condition of (5.9),

$$
\begin{gathered}
\ell_{\mathrm{b}}^{M}(\psi(b))=\ell_{\mathrm{b}}^{N}(b) \text { and } \\
\phi\left(\theta_{\mathrm{in}}^{N}(i, b)\right)=\theta_{\mathrm{in}}^{M}(i, \psi(b)) \text { and } \phi\left(\theta_{\text {out }}^{N}(b, j)\right)=\theta_{\text {out }}^{M}(\psi(b), j) \text { for all } i, j,
\end{gathered}
$$

is satisfied for all $b \in B^{N}$. This side condition amounts precisely to conditions (5.2), (5.3), and (5.4) in the definition of isomorphism. Moreover, the requirement that $\phi$ (viewed as a function from $W^{N}$ to $W^{M}$ ) is an indexing is equivalent to condition (5.1). By Lemma 5.4, these conditions are necessary and sufficient for the pair $(\phi, \psi)$ to be an isomorphism of diagrams.

Proof of Lemma 4.1. We have already noted that the right-to-left direction is trivial. For the left-to-right direction, note that we just showed that the polynomial $\llbracket N \rrbracket_{M}$ has a nonzero coefficient at $\prod_{b \in B^{M}} \xi_{b}$ if and only if there exists an isomorphism between $N$ and $M$. In particular, $\llbracket M \rrbracket_{M}$ has a non-zero such coefficient. Therefore, if $\llbracket N \rrbracket_{M}=\llbracket M \rrbracket_{M}$, then $\llbracket N \rrbracket_{M}$ has a non-zero such coefficient, and it follows that $N$ and $M$ are isomorphic diagrams.

We note that the proof of Lemma 4.1 yields a stronger property:

Corollary 5.9. The coefficient of $\llbracket N \rrbracket_{M}$ at the monomial $\prod_{b \in B^{M}} \xi_{b}$ is equal to the number of different isomorphisms from $N$ to $M$. 
5.5. Proof of the main result. The following is an immediate consequence of Lemma 4.1.

Proposition 5.10. Let $M, N$ be two simple, closed terms in the language of dagger traced monoidal categories. Suppose that $\llbracket M \rrbracket=\llbracket N \rrbracket$ for every possible interpretation in finite dimensional Hilbert spaces. Then $M=N$ holds in the graphical language.

The proof of Theorem 2.2 then consists of removing the conditions "simple", "closed", and "in the language of dagger traced monoidal categories", using the reductions outlined in Section 3. We give the details here.

Lemma 5.11. The condition "simple" can be removed from Proposition 5.10 ,

Proof. Let $M, N: A \rightarrow B$ be two terms, not necessarily simple. It suffices to show that if there is some object variable $A$ such that $M$ and $N$ have a different number of trivial cycles of type $A$, then there exists an interpretation such that $\llbracket M \rrbracket \neq \llbracket N \rrbracket$. Let $\llbracket A \rrbracket$ be a 2 -dimensional Hilbert space with basis $\{a, b\}$. For any $B \neq A$, let $\llbracket B \rrbracket$ be a 1-dimensional Hilbert space with basis $\{c\}$. We call the basis vectors $a$ and $c$ distinguished. For a morphism variable $f: \boldsymbol{A} \rightarrow \boldsymbol{B}$, define

$$
\llbracket f \rrbracket_{w_{1}, \ldots, w_{n}}^{w_{1}^{\prime}, \ldots, w_{m}^{\prime}}=\left\{\begin{array}{l}
1 \text { if } w_{1}, \ldots, w_{n}, w_{1}^{\prime}, \ldots, w_{m}^{\prime} \text { are all distinguished, } \\
0 \text { otherwise }
\end{array}\right.
$$

It is then easy to see that any simple closed term $P$ satisfies $\llbracket P \rrbracket=1$. Namely, in (5.6), $\prod_{b \in B^{N}} b(\phi)$ is equal to 1 precisely for the unique indexing $\phi \in \operatorname{Idx}^{N}$ that picks only distinguished basis vectors, and 0 otherwise. Also, the interpretation of a trivial cycle of type $A$ is $\operatorname{Tr}\left(\operatorname{id}_{A}\right)=\operatorname{dim} \llbracket A \rrbracket=2$, whereas the interpretation of any other trivial cycle is $\operatorname{dim} \llbracket B \rrbracket=1$. Therefore, if $M$ and $N$ have $k$ and $l$ trivial cycles of type $A$, respectively, then $\llbracket M \rrbracket=2^{k}$ and $\llbracket N \rrbracket=2^{l}$, which implies $\llbracket M \rrbracket \neq \llbracket N \rrbracket$ if $k \neq l$.

Lemma 5.12. The condition "closed" can be removed from Proposition 5.10.

Proof. Suppose Proposition 5.10 holds for all closed terms. Let $M, N: A \rightarrow B$ be two terms that are not necessarily closed, such that every possible interpretation satisfies $\llbracket M \rrbracket=\llbracket N \rrbracket$. We can extend the language with two new morphism variables $f: I \rightarrow A$ and $g: B \rightarrow I$, and apply the proposition to the terms $M^{\prime}=g \circ M \circ f$ and $N^{\prime}=g \circ N \circ f$. It follows that the diagrams for $g \circ M \circ f$ and $g \circ N \circ f$ are isomorphic in the graphical language. But since $f, g$ are new symbols that do not occur in $M$ and $N$, this implies that $M$ and $N$ are isomorphic as well.

Lemma 5.13. Proposition 5.10 holds for terms in the language of dagger compact closed categories, over any signature of dagger compact closed categories.

Proof. Let $\Sigma$ be a signature of dagger compact closed categories. This means that the domain and codomain of each morphism variable is a sequence of object variables and their duals, for example $f: A^{*} \otimes B \otimes C^{*} \rightarrow D^{*} \otimes E$. Let $\mathcal{C}_{\Sigma}$ be the free dagger compact closed category over $\Sigma$. The first observation is that there exists a signature $\Sigma^{\prime}$ of dagger monoidal categories such that $\mathcal{C}_{\Sigma^{\prime}} \cong \mathcal{C}_{\Sigma}$ are isomorphic categories. Indeed, $\Sigma^{\prime}$ is obtained from $\Sigma$ by replacing each $A^{*}$ in the domain of a morphism variable by $A$ in its codomain, and vice versa; for example, the above $f$ in $\Sigma$ will be replaced by $f^{\prime}: B \otimes D \rightarrow A \otimes C \otimes E$ in $\Sigma^{\prime}$. Then $\mathcal{C}_{\Sigma^{\prime}} \cong \mathcal{C}_{\Sigma}$ because $f$ and $f^{\prime}$ are interdefinable in any compact closed category.

What we must show is that for all $f \neq g: A \rightarrow B$ in $\mathcal{C}_{\Sigma}$, there exists some dagger compact closed functor $F: \mathcal{C}_{\Sigma} \rightarrow$ FinHilb such that $F(f) \neq F(g)$. Equivalently, we have 
to show that there exists a faithful dagger compact closed functor $\bar{F}: \mathcal{C}_{\Sigma} \rightarrow$ FinHilb $^{X}$ into some discrete power FinHilb ${ }^{X}=\prod_{i \in X}$ FinHilb of the category of finite dimensional Hilbert spaces.

Let $\mathcal{T}_{\Sigma^{\prime}}$ be the free dagger traced symmetric monoidal category over $\Sigma^{\prime}$. It is an easy exercise to prove that Joyal, Street, and Verity's "Int"-construction 44 freely embeds any dagger traced symmetric monoidal category $\mathbf{D}$ in a dagger compact closed category $\operatorname{Int}(\mathbf{D})$, and moreover, that any faithful dagger traced monoidal functor $G: \mathbf{D} \rightarrow \mathbf{C}$ into a dagger compact closed category extends to a faithful dagger compact closed functor $\hat{G}: \operatorname{Int}(\mathbf{D}) \rightarrow$ C. Applying this to the situation where $\mathbf{D}=\mathcal{T}_{\Sigma}$ and $\mathbf{C}=\mathcal{C}_{\Sigma^{\prime}}$, and using the respective universal properties of $\mathcal{C}_{\Sigma^{\prime}}$ and of the Int-construction, we obtain an equivalence of dagger compact closed categories $\mathcal{C}_{\Sigma^{\prime}} \simeq \operatorname{Int}\left(\mathcal{T}_{\Sigma}\right)$.

By hypothesis (i.e., Proposition 5.10, with the conditions "simple" and "closed" already removed), there exists a faithful traced monoidal functor $H: \mathcal{T}_{\Sigma^{\prime}} \rightarrow$ FinHilb ${ }^{X}$ for some $X$. Then the composition

$$
\bar{F}:=\mathcal{C}_{\Sigma} \stackrel{\cong}{\rightarrow} \mathcal{C}_{\Sigma^{\prime}} \stackrel{\simeq}{\rightarrow} \operatorname{Int}\left(\mathcal{T}_{\Sigma}\right) \stackrel{\hat{H}}{\rightarrow} \text { FinHilb }^{X}
$$

is the desired faithful dagger compact closed functor.

\section{Generalizations}

Other rings and fields. The result of this paper (Theorem 2.2) can be adapted to other fields besides the complex numbers. It is true for any field $k$ of characteristic 0 with a non-trivial involutive automorphism $x \mapsto \bar{x}$. (Non-trivial means that for some $x, \bar{x} \neq x$ ).

The only special property of $\mathbb{C}$ that was used in the proof, and which may not hold in a general field $k$, was the existence of transcendentals. This problem is easily solved by first considering the field of fractions $k\left(x_{1}, \bar{x}_{1} \ldots, x_{n}, \bar{x}_{n}\right)$, where the required transcendentals have been added freely. The proof of Lemma 4.1 then proceeds without change. Finally, once an interpretation over $k\left(x_{1}, \bar{x}_{1} \ldots, x_{n}, \bar{x}_{n}\right)$ has been found such that $\llbracket M \rrbracket \neq \llbracket N \rrbracket$, we use the fact that in a field of characteristic 0 , any non-zero polynomial has a non-root. Thus we can instantiate $x_{1}, \ldots, x_{n}$ to specific elements of $k$ while preserving the inequality $\llbracket M \rrbracket \neq \llbracket N \rrbracket$. Note that therefore, Theorem 2.2 holds for the given field $k$; however, Lemma44.1 only holds for $k\left(x_{1}, \bar{x}_{1} \ldots, x_{n}, \bar{x}_{n}\right)$.

Moreover, the elements $x_{1}, \ldots, x_{n}$ of the preceding paragraph can always be instantiated to integers; therefore, the results also hold if one replaces $k$ by the ring of Gaussian integers $k=\mathbb{Z}[i]$. In elementary terms: if a certain equation fails to hold in dagger compact closed categories, then one can always find a counterexample in matrices with entries of the form $a+b i$, where $a, b \in \mathbb{Z}$. Moreover, $i=\sqrt{-1}$ can be replaced by $\sqrt{d}$ for any non-square integer $d$.

Non-equational properties. The completeness result of this paper applies to properties expressible as equations. One may ask whether it can be generalized to other classes of properties, such as implications, Horn clauses, or more general logical formulas. Unfortunately, this is not the case; there exist (universally quantified) implications that hold in FinHilb, but fail to hold in arbitrary dagger compact closed categories. One example of such an implication is

$$
\forall f, g: A \rightarrow B \quad\left(f^{\dagger} f f^{\dagger} f=g^{\dagger} g g^{\dagger} g \quad \Rightarrow \quad f^{\dagger} f=g^{\dagger} g\right) .
$$


This is true in FinHilb, because each hermitian positive operator has a unique hermitian positive square root. But it fails in general dagger compact closed categories. Perhaps the simplest counterexample is the ring $\mathbb{Z}_{5}$, regarded as a one-object dagger compact closed category with composition and tensor of morphisms given by ring multiplication, and dagger given by the identity operation. Taking $f=1$ and $g=2$, the premise is satisfied and the conclusion is not.

Bounded dimension. The interpretation $\llbracket-\rrbracket_{M}$ from the proof of Lemma 4.1 uses Hilbert spaces of unbounded finite dimension. One may ask whether Theorem 2.2 remains true if the interpretation of object variables is restricted to Hilbert spaces of some fixed dimension $n$. This is known to be false when $n=2$. Here is a counterexample due to Bob Paré: the equation $\operatorname{tr}(A A B B A B)=\operatorname{tr}(A A B A B B)$ holds for all $2 \times 2$-matrices, but does not hold in the graphical language. Indeed, by the Cayley-Hamilton theorem, $A^{2}=\mu A+\nu I$ for some scalars $\mu, \nu$. Therefore

$$
\begin{aligned}
& \operatorname{tr}(A A B B A B)=\mu \operatorname{tr}(A B B A B)+\nu \operatorname{tr}(B B A B), \\
& \operatorname{tr}(A A B A B B)=\mu \operatorname{tr}(A B A B B)+\nu \operatorname{tr}(B A B B),
\end{aligned}
$$

and the right-hand-sides are equal by cyclicity of trace. It is not currently known to the author whether Theorem 2.2 is true when restricted to spaces of dimension 3 .

\section{ACKNOWLEDGMENTS}

Thanks to Gordon Plotkin for telling me about this problem and for discussing its solution. Thanks to Bob Paré for the counterexample in dimension 2.

\section{REFERENCES}

[1] S. Abramsky and B. Coecke. A categorical semantics of quantum protocols. In Proceedings of the 19th Annual IEEE Symposium on Logic in Computer Science, LICS 2004, pages 415-425. IEEE Computer Society Press, 2004.

[2] R. P. Geroch. Special topics in particle physics. Unpublished lecture notes, University of Texas at Austin. Available from http://strangebeautiful.com/other-texts/geroch-qft-lectures.pdf, 1971.

[3] M. Hasegawa, M. Hofmann, and G. Plotkin. Finite dimensional vector spaces are complete for traced symmetric monoidal categories. In Pillars of Computer Science: Essays Dedicated to Boris (Boaz) Trakhtenbrot on the Occasion of His 85th Birthday, Lecture Notes in Computer Science 4800, pages 367-385. Springer, 2008.

[4] A. Joyal, R. Street, and D. Verity. Traced monoidal categories. Mathematical Proceedings of the Cambridge Philosophical Society, 119:447-468, 1996.

[5] S. Lang. Algebra. Springer Graduate Texts in Mathematics 211. 2004.

[6] R. Penrose and W. Rindler. Spinors and Space-Time: Volume 1, Two-Spinor Calculus and Relativistic Fields. Cambridge University Press, 1984.

[7] P. Selinger. Dagger compact closed categories and completely positive maps. In Proceedings of the 3rd International Workshop on Quantum Programming Languages, Electronic Notes in Theoretical Computer Science 170, pages 139-163. Elsevier, 2007.

[8] P. Selinger. A survey of graphical languages for monoidal categories. In B. Coecke, editor, New Structures for Physics, Lecture Notes in Physics 813, pages 289-355. Springer, 2011.

[9] R. M. Wald. Quantum Field Theory in Curved Spacetime and Black Hole Thermodynamics. University Of Chicago Press, 1994.

This work is licensed under the Creative Commons Attribution-NoDerivs License. To view a copy of this license, visit http://creativecommons.org/licenses/by-nd/2.0/ or send a letter to Creative Commons, 171 Second St, Suite 300, San Francisco, CA 94105, USA, or Eisenacher Strasse 2, 10777 Berlin, Germany 